\title{
Iatrogenic nerve injuries
}

\author{
J. B. WINER \\ M.R.C.P. \\ M. J. G. HARRISON \\ D.M., F.R.C.P. \\ Department of Neurological Studies, Middlesex Hospital, Mortimer Street, London, W.1.
}

\section{Summary}

Thirty-one examples of iatrogenic peripheral nerve injuries have been collected from a review of the case records of one neurological referral centre over a 7 year period. The clinical details are described to call attention to the special care needed with the management of patients subjected to certain invasive procedures.

\section{Introduction}

The importance of drug therapy as a cause of peripheral neuropathy is well appreciated. However, the risks of iatrogenic isolated traumatic nerve injuries have been less well documented.

The authors present a review of the iatrogenic peripheral nerve injuries referred for electromyography (EMG) and assessment of nerve conduction to the Department of Neurological Studies of the Middlesex Hospital over the last seven years. The Department provides an EMG service for a number of hospitals outside the Middlesex group, so the true frequency of these problems cannot be simply calculated.

\section{Method}

The EMG department records for the years 1974-1981 were reviewed, and all cases referred with peripheral nerve injuries were extracted. The notes of all these patients were reviewed, and only those whose nerve lesions were due to medical intervention were included. A number of patients sustained nerve damage as a result of operations in which such damage is an obvious and well recognized risk, such as facial nerve involvement after the removal of acoustic neuromas, and parotid tumours. These were not included in the study.

An effort was made to exclude any patient who might have an inherited predisposition to pressure sensitivity of peripheral nerves, as has been reported in some families (Earl et al., 1964). During the 7year period of the study, one case of radial and one of an ulnar nerve injury were seen in such families. Care was further taken to exclude underlying peripheral neuropathies from the study by including more than one nerve in the EMG examination in each case.

\section{Results}

The distribution of nerves involved in 31 cases of iatrogenic nerve injury is shown in Table 1.

TABLE 1. The distribution of nerves involved in 31 cases of iatrogenic injury

\begin{tabular}{llll}
\hline Upper limb $(n=16)$ & & Lower limb $(n=15)$ & \\
Median nerve & 6 & Sciatic nerve & 5 \\
Ulnar nerve & 4 & Lateral popliteal nerve & 4 \\
Brachial plexus/root & 4 & Femoral nerve & 2 \\
Tourniquet paralysis & 1 & Inguinal nerve & 20 \\
Digital nerve & 1 & Posterior tibial nerve & Sural nerve \\
& & &
\end{tabular}

\section{Median nerve}

Four examples of median nerve damage occurred as a result of cardiac catheterization. The patients had often complained of local pain at the site of catheterization radiating down the arm. On one occasion the local anaesthetic was mistakenly injected directly into the nerve, and in another a large haematoma was thought to be responsible. The following case is described to illustrate the type of injury involved.

An 18-year-old Ugandan had left heart catheterization and coronary arteriography for investigation of a severely abnormal electrocardiogram (ECG). A high brachial artery division was encountered and catheterization of the radial branch produced spasm; the ulnar branch was eventually used for the procedure. The day following catheterization the patient noticed some slight sensory loss in the median territory of the hand, with severe weakness of the median supplied muscles of the hand and forearm. There was some improvement in the weakness over the next few days but the patient discharged herself and no follow-up was possible.

A fifth patient was referred for EMG studies as an aid to the diagnosis of Friedrich's ataxia. She also suffered from a cardiomyopathy and had been investigated by coronary arteriography in the past 
with no complications. There were no symptoms of median nerve damage but nerve conduction studies suggested that damage had occurred at the time of catheterization.

A further example of median nerve damage occurred after venesection.

A 25-year-old male had blood taken from the right antecubital fossa without undue pain or distress. The next day he developed tingling in the fingers of the right hand excluding the little finger. There was no bruising 10 days later but deep pressure over the venepuncture site increased the tingling felt in the fingers. Electrical studies showed a local conduction block in the right median nerve.

\section{Ulnar nerve}

Four ulnar nerve injuries occurred postoperatively. In one, a 43-year-old male had a saphenous vein bypass graft to the left anterior descending coronary artery. Five days after the operation when the patient was transferred from intensive care to the general ward a left ulnar nerve lesion was noted which clinically was localizable to the elbow. The EMG findings were suggestive of neuropraxia with no denervation and preservation of the ulnar sensory action potential. The notes make no mention of the lesion which presumably had recovered completely when the patient was seen for review a year later. Another lesion occurred after cardiac surgery, and the third following a period of immobilization in a seriously ill male who developed complications following the drainage of an appendix abscess. A fourth lesion occurred following surgery for ureteric stones.

\section{Cervical roots and brachial plexus}

Three brachial plexus lesions and one cervical root injury were seen.

One patient with her arm extended during a nephrectomy developed a traction injury of the C8 and T1 roots with weakness of the long flexors and interossei with sensory loss over the inner aspect of the arm.

A second patient sustained a traction injury during a right modified mastectomy for carcinoma of the breast. Postoperatively there was severe weakness of the deltoid, biceps, brachialis, brachioradialis, and wrist extensors with milder weakness of finger flexion and extension. Over the next two weeks there was improvement in wrist extension and the patient was followed-up at another hospital. A brachial plexus injury at surgery was considered to be the cause. A third patient noticed weakness of the right hand one week after a posterior spinal fusion for scoliosis. There was global arm weakness with absent reflexes and, again, a brachial plexus injury at the time of surgery was blamed.
The cervical root injury occurred following an operation for bilateral cervical ribs in a patient who complained of paraesthesiae in the fingers of both hands. Immediately following the operation she developed numbness overlying the left shoulder blade and a left $\mathrm{C} 5$ root lesion with weakness of the muscles supplied by this root. The sensory loss persisted and, on review 6 years later, there was also persistent slight weakness of the rhomboids and spinati.

\section{Tourniquet paralysis}

The following example of a tourniquet paralysis was seen.

A 58-year-old female fractured her right radius and ulnar in February 1981. The fracture was plated with the use of a tourniquet during the procedure. Two days following the operation tone was found to be flaccid in the forearm with a paralysis involving the median radial and ulnar nerves. There was almost complete paralysis at the wrist with considerable improvement on review in one month. However, thumb and finger movement was still impaired.

\section{Digital nerve}

A 77-year-old man complained of troublesome paraesthesiae of his left index finger since exploration of it for a foreign body in a casualty department. On examination there was a sensory loss over the distribution of one digital nerve. Nerve conduction studies supported the clinical view that only one digital nerve was involved.

\section{Sciatic nerve}

Five examples of sciatic nerve damage were seen and also one example of a posterior tibial lesion following damage to the sciatic nerve high in the buttock. On one occasion the nerve was damaged as a result of a pressure palsy following a therapeutic dose of barbiturate sleeping tablets. One lesion followed the application of skeletal traction and plaster to a compound fracture of the left femur. A lateral popliteal palsy may also have been present secondary to pressure from the plaster cast. A further lesion was noticed in a comatose patient nursed in the intensive care unit for a 10-day period of unconsciousness, and one followed a Thompson arthroplasty for a right sub-capital fracture of the femur. The following case illustrates the typical history obtained.

A 38-year-old female was operated upon for primary lymphoedema of the left leg. Immediately she recovered consciousness she was noticed to have a left foot drop. EMG studies suggested a sciatic nerve lesion involving both lateral popliteal and posterior tibial divisions. One year following the 
operation there was no evidence of re-inervation either clinically or on EMG.

\section{Femoral nerve}

Two examples of femoral nerve injury were seen. One occurred after the use of anticoagulants.

A 65-year-old female was admitted with a 6month history of amaurosis fugax in the left eye. There was a left carotid bruit and a reduced left carotid pulse. She was anticoagulated with heparin, and carotid angiography performed. Three days after the angiogram which involved catheterization of the femoral artery she developed a painful femoral neuropathy with an absent right knee jerk and some weakness of the right quadriceps. She had a left carotid endarterectomy performed without complications and the femoral neuropathy resolved with time.

The second example occurred following the repair of a vesico-vaginal fistula. The nerve was damaged above the inguinal ligament and electromyography showed that Wallerian degeneration had occurred. A haematoma was thought to be responsible but at explorative operation 8 weeks later the nerve was found to be intact without evidence of pressure from a blood clot. Considerable improvement had occurred at review 7 months later.

\section{Lateral popliteal nerve}

Four cases of lateral popliteal nerve lesions were seen. One occurred following the excision of a haemangioma of the left leg involving resection of the middle two-thirds of the fibula. Another occurred following a repeat right upper tibial osteotomy and involved only the part of the nerve supplying extensor hallucis longus. Unfortunately in this case it was impossible to get EMG confirmation of the lesion acutely because the leg was placed in a plaster cast postoperatively. However the clinical findings were unequivocal.

In the third case the lesion was noted immediately after operation for intermittent claudication of the left leg. A left femoral popliteal bypass graft was performed but the patient had to be taken back to theatre following a haemorrhage from the site of the anastomosis and after the second operation a left foot drop developed. There was some sensory loss over the anterior aspect of the foot as well as weakness of dorsiflexion and eversion of the left foot. EMG suggested an incomplete lesion and there was considerable improvement over the next two weeks.

The last case occurred after traction was applied to the leg following a hip operation. There was no recovery at 4 months.

\section{Sural nerve}

A 46-year-old female with a 6-month history of pain and numbness in the distribution of the right sural nerve, had had a prominent right calcaneum trimmed in 1958 and postoperative scar tissue was thought to have traumatized her sural nerve over the years. Nerve conduction studies confirmed a right sural nerve lesion and the patient had a surgical decompression. The nerve was found to be embedded in scar tissue and a great deal of improvement of symptoms occurred postoperatively.

\section{Inguinal nerve}

A 70-year-old male developed left-sided groin pain radiating to the left side of the scrotum following a repeat left hernia operation in 1975. Excision of the herniorraphy scar in July 1979 rendered the patient free of pain although some discomfort recurred over the next year. It seemed that entrapment of the left inguinal nerve in the scar tissue was responsible for the original symptoms.

A second case of inguinal nerve damage post herniorraphy was seen in which the symptoms of numbness of the right groin and inner aspect of the right thigh started immediately postoperatively. In this case there must have been damage at operation.

\section{Discussion}

The majority of nerve injuries described occurre as a result of surgery or from faulty body positiof during operation. In this way some relatively triviat procedures were able to produce a severe neurological disability.

The largest number of injuries involved the median nerve and most occurred during cardiac catheterization. In 1960, Schneck described a case of anterior interosseus nerve damage following catheterization and there have been reports of median nerve damage following arterial catheterization for blood gas estimation (Patten, 1969; Macow and Furtrell, 1973; Luce et al., 1976) or blood pressure monitoring (Little, 1976). Haematoma rather than direct damage to the nerve is often responsible. The authors' case of asymptomatic median nerve damage following cardiac angiography is of particular interest since it suggests that the incidence of median nerve damage during this procedure is far greater than is generally appreciated.

The sciatic and the common peroneal nerves were the two other nerves most commonly damaged. Hip surgery is often responsible (Campbell et al., 1960; Rodriguez, Austin and McBride, 1964) but any surgical procedure in the upper thigh or buttock can place the sciatic nerve at risk.

No example of sciatic nerve damage as a result of misplaced injection was seen and it appears that the dangers of this are well publicized and appreciated.

Ulnar nerve injuries and brachial plexus lesions 
almost certainly occurred as a result of faulty body position either with the unconscious surgical patient or the ventilated patient in the intensive care unit. Lincoln and Sawyer (1961) and Seddon (1975) review the common injuries involved and suggest ways in which they can be avoided.

The case of ilio-inguinal nerve injury as a result of nerve entrapment post herniorraphy illustrates how some iatrogenic lesions can occur some years after the operation. Schneck (1960) described one case of ilio-inguinal entrapment also occurring after herniorraphy in which the symptoms did not begin until 16 years after the original operation.

While the very nature of some operations must always place anatomically vulnerable nerves at risk it is obvious that heightened awareness of the potential for iatrogenic nerve trauma would help reduce the prevalence of such problems. Physicians involved in the performance of invasive procedures as well as surgeons need to be aware of these risks.

\section{References}

Campbell, R.D., Mason, J.B., Wilson, P.D. \& Wade, P.A. (1960) The use of intramedullary prosthetic replacement in fractures of the femoral neck. American Journal of Surgery, 99, 745.

Earl, C.J., Fullerton, P.M., Wakefield, G.S. \& Schutta, H.S. (1964) Hereditary neuropathy with liability to pressure palsy. Quarterly Journal of Medicine, 33, 581.

LINCOLN, J.R. \& SAWYER, H.P. (1961) Complications related to body positions. Anaesthiology, 22, 804.

LitTle, W.A. (1976) Median nerve palsy-a complication of brachial artery cannulation. Postgraduate Medical Journal, $7,110$.

Luce, E.A., Furtrell, J.W., Shaw Wilges, E.F. \& Hoopes, J.E. (1976). Compression neuropathy following brachial artery puncture on anticoagulated patients. Journal of Trauma, 16, 717.

Macow, W.C. \& Furtrell, J.W. (1973) Median nerve neuropathy after percutaneous puncture of the brachial artery in patients receiving anticoagulants. New England Journal of Medicine, 288, 1396.

Patten, B.M. (1969) Neuropathy induced by haemorrhage. Archives of Neurology, 21, 381.

Rodriguez, M.J., Austin, E. \& McBride, F.J. (1964) Peroneal nerve damage following insertion of Austin Moore prosthesis. Archives of Physical Medicine, 45, 283.

SCHNeCK, S.A. (1960) Peripheral and cranial nerve injuries resulting from general surgical procedures. Archives of Surgery, 81, 855.

Seddon, Sir Herbert (1975) Surgical Disorder of Peripheral Nerves. Churchill Livingstone, Edinburgh. 